\title{
USO DE UM SISTEMA DE INFORMAÇÃO EM UMA INSTITUIÇÃO PÚBLICA um estudo de caso
}

\section{1- Eliete dos Reis*}

Mestre em Administração pela Universidade Federal de Santa Maria (UFSM), Brasil. elietedosreis@gmail.com

http://lattes.cnpq.br/4996862852253906

\section{2- Mauri Leodir Löbler}

Doutorado em Administração pela Universidade Federal do Rio Grande do Sul (UFRGS), Brasil. Professor Adjunto da Universidade Federal de Santa Maria (UFSM), Brasil. lobler@ccsh.ufsm.br

http://lattes.cnpq.br/7320669188854401

\section{3- Simone Alves Pacheco de Campos}

Doutoranda em Administração pela Universidade Federal do Rio Grande do Sul (UFRGS), Brasil. simoneapcampos@gmail.com

http://lattes.cnpq.br/2403565845137368

\section{4- Márcio Sampedro Ramos}

Mestrando em Administração pela Universidade Federal de Santa Maria (UFSM), Brasil. ramos_marcio@yahoo.com.br

http:///attes.cnpq.br/0166839185391727 


\section{USO DE UM SISTEMA DE INFORMAÇÃO EM UMA INSTITUIÇÃO PÚBLICA: UM ESTUDO DE CASO}

\section{RESUMO}

Como resultado do avanço tecnológico e da velocidade com que as informações são processadas, a cada momento a sociedade passa a dispor de novas tecnologias, desenvolvidas com o propósito de facilitar o trabalho dos indivíduos. Frente a este cenário, este estudo teve como objetivo identificar os fatores que explicam o uso de um sistema de informação, a partir dos modelos de aceitação à tecnologia, TAM e TTF, considerados consenso na literatura desse tema. Assim, realizou-se uma pesquisa, através de uma abordagem qualitativa e descritiva, por meio desses modelos que, na maioria dos casos, são aplicados em análises de natureza quantitativa. Com relação ao método para realização desta pesquisa, utilizou-se um estudo de caso em uma instituição pública. A análise dos resultados obtidos demonstrou que todos os fatores avaliados, ou seja, utilidade percebida, facilidade de uso, intenção de uso e ajuste da tecnologia à tarefa explicaram o uso do sistema estudado. Desta forma, percebe-se que o uso de um SI não está relacionado somente com o ajuste entre a tarefa e a tecnologia, ou com o comportamento e as atitudes dos indivíduos frente aos sistemas, mas também com a combinação desses fatores (Klopping e McKinney, 2004).

\section{Palavras-chave}

Sistema de informação, Uso, Modelos de aceitação

\section{USE OF AN INFORMATION SYSTEM IN A PUBLIC INSTITUTION: A CASE STUDY}

\section{ABSTRACT}

As a result of technological advances and the speed at which information is processed, every moment society starts to count on new technologies developed for the purpose of making the work of individuals easier. Concerning this scenario, this study had the objective to identify the factors which explain the use of a managerial information system from the technology acceptance models, TAM and TTF, considered a consensus in the literature about the theme. Thus, a research of qualitative and descriptive approach was conducted by means of these models which, in most cases, are applied in quantitative analysis. Relating to the method for carrying out this research, a case study was used in a public institution. The analysis of the results which were obtained showed that all the factors evaluated, that is, noticeable utility, facility of use, intention of use and adjustment of technology to task were relevant to explain the use of the system being studied. In this way, it is noticed that the use of an Information System is not only related to the adjustment between task and technology, or to the individuals' behavior and attitude toward the systems, but also to the combination of these factors (Klopping \& McKinney, 2004).

\section{Keywords}

Information System; Use; Acceptance models. 


\section{Introdução}

Com o avanço tecnológico e com a velocidade em que as informações são processadas, a cada momento, a sociedade passa a dispor de novas tecnologias, desenvolvidas com o propósito de facilitar o trabalho dos indivíduos. No caso das organizações, a maioria faz uso de sistemas de informações a fim de que as informações precisas e necessárias estejam disponíveis quando necessário.

Segundo Chau e Hu (2001), o recente desenvolvimento da Tecnologia da Informação - TI e suas aplicações por profissionais altamente especializados tem-se multiplicado substancialmente, o que justifica a importância de considerar teorias que podem explicar ou prever a aceitação ao uso da tecnologia.

Embora não exista um consenso na literatura acerca da definição de tecnologia da informação (TI), Turban, Rainer e Potter (2005) a definem num sentido mais amplo, descrevendo-a como o conjunto de recursos de informação da organização, seus usuários e os gerentes que os supervisionam, incluindo ainda a infraestrutura de TI e todos os demais sistemas de informação na organização.

No que se refere aos sistemas de informação, de acordo com Turban, Rainer e Potter (2005, p. 40) um "sistema de informação é um processo que coleta, processa, armazena, analisa e dissemina informações para uma finalidade específica". Neste sentido, os sistemas de informação são eficientes aplicativos, que têm sido desenvolvidos para otimizar os fluxos de informação relevantes no âmbito de uma organização, desencadeando um processo de conhecimento e de tomada de decisão e intervenção na realidade (MORESI, 2000). Neste contexto, salienta-se que, neste estudo, os termos Tecnologia de Informação e Sistema de Informação (SI) são tratados como sinônimos.

Devido à importância que os SI representam para as organizações, Zhang, Li e Sun (2006) afirmam que a identificação do uso da tecnologia pelos seus usuários e os comportamentos desses em relação a mesma tornaram-se prioridade. Tal fato pode ser comprovado através da crescente literatura de sistemas de informação sobre aceitação de SI explicados pelos modelos significativos que descrevem o comportamento dos usuários.

Diante desse cenário, Agarwal e Prasad (1998) acrescentam que alguns modelos têm sido desenvolvidos para melhor entender e explicar as atitudes e comportamentos individuais relacionados à Tl: o Modelo da Teoria do Comportamento Planejado (Ajzen, 1985, 1988; Ajzen e Madden, 1986); o Modelo da Aceitação da Tecnologia (TAM) (Davis, 1989; Davis, Bagozzi, \& Warshaw, 1989); o Modelo Ajuste TarefaTecnologia (TTF) (Goodhue, 1995), entre outros. Dessa forma, alguns estudos vêm trabalhando com a combinação desses modelos, como é o caso de Dishaw e Strong (1999);Klopping e Mckinney (2004).

Segundo Dishaw, Strong e Bandy (2002), os dois modelos mais frequentemente utilizados para descrever a aceitação de um sistema de informação são os modelos TAM (Davis, 1989) e TTF (Goodhue, 1995). O Modelo TAM aborda dois construtos: a utilidade percebida e a facilidade de uso percebida, analisando como a percepção de utilidade e facilidade de uso influencia na intenção de uso de um sistema de informação e no desempenho do usuário. Já o modelo TTF está baseado no construto da capacidade do SI para apoiar uma tarefa, ou seja, busca identificar como a adequação entre características das tarefas dos usuários e da tecnologia pode influenciar positivamente no desempenho do usuário.

Estes dois importantes modelos, TAM (Davis 1989) e TTF (Goodhue 1995), foram combinados por Dishaw, Strong e Bandy (2002), porque capturam dois diferentes aspectos de escolha para aceitação de SI. A atitude e comportamento sob os quais o modelo TAM é baseado assinalam que as crenças e atitudes dos usuários em direção a um SI são amplamente determinadas se os usuários apresentarem comportamento de uso de um SI. O modelo TTF decididamente adota uma abordagem racional, assumindo que os usuários optem por utilizá-lo porque proporciona vantagens, tais como melhoria no desempenho profissional, independentemente de suas atitudes em relação ao SI (Goodhue, 1995).

Neste contexto, tendo em vista a importância dos sistemas de informação na realizada organizacional, uma vez que, os mesmos estão alterando de maneira significativa as relações produtivas, os fluxos de trabalho, as tomadas de decisão e o desenvolvimento das estratégias competitivas (Dias, 2006), emerge a seguinte inquietação que motivou a realização desta pesquisa: Quais os fatores que motivam um indivíduo usar um sistema de informação?

Neste escopo, o objetivo geral deste estudo está centrado em identificar quais os fatores que explicam o uso de um sistema de informação. Visando atingir o objetivo proposto, este estudo teve seu foco de análise voltado a uma organização do setor público, situada na região central do estado do Rio Grande do 
Sul. Desta forma, foram definidos os seguintes objetivos específicos que balizaram a realização desta pesquisa: (i) identificar o uso do SI através da facilidade, utilidade percebida e intenção de uso; e (ii) investigar o uso de um SI através do ajuste às tarefas.

A relevância do presente estudo está em compreender os fatores que explicam o comportamento do uso dos sistemas de informação a partir de modelos que são considerados consenso na literatura desse tema. Cabe ressaltar que a utilização de mais de um modelo amplia o nível de explicação a respeito do uso de um sistema de informação. Ainda, sob a ótica de contribuições, esta pesquisa traz à luz informações sobre a adequação entre tarefa e tecnologia e sobre suas analogias com o desempenho do usuário. Dessa maneira, releva aspectos que podem contribuir para maximização da utilização dos SI.

Para atingir o objetivo proposto, o trabalho está estruturado em mais cinco seções, além da introdução: na seção dois, aborda-se o referencial teórico que balizou a realização deste estudo. Na seção três, é apresentada a arquitetura metodológica que possibilitou a operacionalização da pesquisa. Na seção quatro, são apresentados os resultados alinhados com os objetivos propostos. Na seção cinco, abordam-se as considerações finais seguidas das referências bibliográficas utilizadas no estudo em questão.

\section{Referencial Teórico}

\subsection{Sistemas de Informação}

Dentre os progressos empresariais que tem proporcionado ganhos consideráveis na eficiência dos processos administrativos, está o uso dos Sistemas de Informação (SI). De acordo com Moresi (2000), os sistemas de informação têm sido desenvolvidos para otimizar os fluxos de informação relevantes no âmbito de uma organização, desencadeando um processo de conhecimento e de tomada de decisão e intervenção na realidade.

Laudon e Laudon (2004) definem que, a partir da perspectiva de uma empresa, um SI é uma solução organizacional e administrativa e que utiliza a tecnologia de informação para enfrentar os desafios propostos pelo ambiente. Dessa forma, o gestor necessita conhecer as dimensões mais amplas da organização, da administração e da tecnologia de informação dos sistemas e sua capacidade de fornecer soluções para os desafios e problemas no âmbito empresarial.

Neste contexto, os sistemas de informações são considerados como um conjunto de componentes interligados, que fazem a coleta, o processamento, o armazenamento, e a distribuição de informações que auxiliam na execução das atividades da empresa (Laudon e Laudon, 2004; Stair e Reynolds, 2002; Audy, Andrade e Cidral, 2007). Para Turban et. al. (2006), um sistema de informação (SI) é um sistema capaz de coletar, processar, armazenar, analisar e disseminar informações para atender um propósito específico. Como qualquer sistema, um SI inclui entradas (dados e instruções) e saídas (relatórios e cálculos) (O'Brien, 2004) e também engloba pessoas, procedimentos e facilidades físicas e opera em um determinado ambiente (Turban, Rainer Jr e Potter, 2005).

Em termos conceituais, os sistemas de informação podem ser classificados de maneira diferente (O'Brien, 2004). Dessa forma, vários tipos de sistemas de informação podem ser classificados, ora como operações, ora como sistemas de informação gerencial.

Os sistemas de apoio a operações produzem uma diversidade de produtos de informação para uso interno e externo e seu principal papel é processar informações eficientemente, controlar processos, apoiar comunicações e atualizar banco de dados. Os sistemas de processamento de transações, controle de processos e colaborativos são exemplos dessa tipologia de sistemas (O'Brien, 2004).

Os sistemas de informação gerencial se concentram em fornecer informações e apoio aos gestores na tomada de decisão. Assim, vários tipos de sistemas de informação apoiam uma série de responsabilidades administrativas do usuário. Dentre eles, os sistemas de informação gerencial, sistemas de apoio à decisão e sistemas de informação executiva (O'Brien, 2004).

No que se refere à definição detalhada das tipologias de sistemas, salienta-se que, neste estudo, há que se definir os sistemas de informação gerencial, tendo em vista que o SI do caso estudado se enquadra nessa classificação. Neste contexto, um sistema de informação gerencial auxilia os administradores a consolidar o tripé básico de sustentação organizacional: qualidade, produtividade e participação. Segundo Oliveira (1993), esse tipo de sistema pode ser definido como um método formal, utilizado pelas 
organizações a fim de tornar disponíveis informações necessárias para facilitar o processo de tomada de decisão.

Na concepção de Audy, Andrade e Cidral (2007), os sistemas de informação gerencial sintetizam, registram e relatam a situação em que se encontram as operações da organização. Esses sistemas atendem, em grande parte, os gerentes de nível tático da organização na forma de relatórios que apresentam indicadores sobre desempenho de uma determinada área.

O propósito básico de um sistema de informação gerencial para Stair e Reynolds (2002) é ajudar a empresa a alcançar suas metas, fornecendo aos seus gerentes detalhes sobre as operações regulares da organização, de forma que possam controlar, organizar e planejar com mais efetividade e com maior eficiência. Neste sentido, segundo Oliveira (1993), os sistemas de informações gerenciais podem, sob determinadas condições, trazer alguns benefícios para as empresas, dentre eles: redução de custos das operações; melhoria na tomada de decisões, por meio do fornecimento de informações mais rápidas e precisas; melhoria na estrutura organizacional, por facilitar o fluxo de informações; redução do grau de concentração de decisões na empresa; melhoria na produtividade, tanto setorial quanto global; melhoria no acesso às informações, propiciando relatórios mais precisos e rápidos, com menor esforço.

\subsection{O Modelo TAM (Technology Acceptance Mode) - Modelo de Aceitação da Tecnologia}

A aceitação, ou a adoção da $\mathrm{TI}$ tem recebido especial atenção no campo acadêmico e empresarial. Consequentemente, modelos e teorias têm sido desenvolvidos para explicar e prever a aceitação à tecnologia, dentre eles, o modelo de aceitação de tecnologia (Technology Acceptance Model - TAM) proposto por Davis (1989) (Teo, Ursavas e Bahçekapili, 2011). Esse modelo é considerado o mais influente e o mais comumente empregado para descrever a aceitação do indivíduo a um sistema de informação (Lee, Kozar e Larsen, 2003).

Desde o seu desenvolvimento, o TAM tem recebido suporte empírico como um modelo válido em vários contextos e em uma variedade de tecnologias, tendo por finalidade explicar como os usuários percebem e usam a tecnologia (Teo, Ursavas e Bahçekapili, 2011). Com base na Teoria da Ação Racionalizada (TRA) de Fishbein e Ajzen, 1975, que descreve como as crenças dos usuários e suas atitudes estão relacionadas às intenções dos indivíduos, o propósito essencial do modelo TAM, especificamente para usuários de sistemas de informação, é prover uma base para mapear o impacto de fatores externos sobre aqueles internos ao indivíduo como as crenças, atitudes e intenções de uso (Costa Filho, Pires e Hernandez, 2007).

A intenção do desenvolvimento do modelo TAM originou-se de um contrato da IBM-Canadá nos meados dos anos 80, para avaliar o potencial de mercado para novos produtos da marca (Davis, 1989). Para o autor, o foco do modelo consiste no por que os usuários aceitam ou rejeitam a tecnologia da informação e em como melhorar esta aceitação. Assim, o propósito do modelo é testar medidas que possam prever e explicar o uso de sistemas de informação.

Deste modo, o modelo TAM (ilustrado na Figura 1) proporciona um suporte para prever e explicar a aceitação de novas tecnologias pelo seu usuário final, através do efeito de dois construtos: a utilidade percebida e a facilidade de uso percebida, sendo que ambos medeiam completamente os efeitos das variáveis externas, como características do sistema, processo de desenvolvimento, treinamento e intenção de uso (Davis, 1989). É importante salientar que os dois constructos foram adaptados a partir das características da inovação de Rogers (2003), sendo equivalentes à vantagem relativa (utilidade percebida) e à complexidade (facilidade de uso percebida) (Costa Filho, Pires e Hernandez, 2007).

O construto da utilidade percebida é descrito no modelo como a medida pela qual a pessoa acredita que a utilização de um SI irá melhorar o seu desempenho no trabalho (Venkatesh e Bala, 2008). Isto inclui diminuir o tempo para fazer o trabalho, e alcançar maior eficiência e precisão (Teo, Luan e Sing, 2008). Para Moore e Benbasat (1991), a utilidade percebida é vista como uma vantagem relativa, isto é, o grau em que uma inovação tecnológica é percebida como superior em comparação à tecnologia antiga que está sendo substituída.

A facilidade de uso percebida é definida como o grau pelo qual a pessoa acredita que usando um SI, estará livre de esforços ao desempenhar suas atividades (Venkatesh e Bala, 2008). Dessa forma, os indivíduos usarão um SI se confiarem que este uso conduzirá a resultados positivos, convergindo na facilidade de uso percebida e na utilidade percebida (Igbaria, livari e Maragahh, 1995). É plausível que, enquanto os usuários acreditarem que um sistema de informação é útil, ele pode ser difícil de usar e, 
contudo, os benefícios de desempenho de uso são compensados pelo esforço de usar o sistema (Davis, 1989).

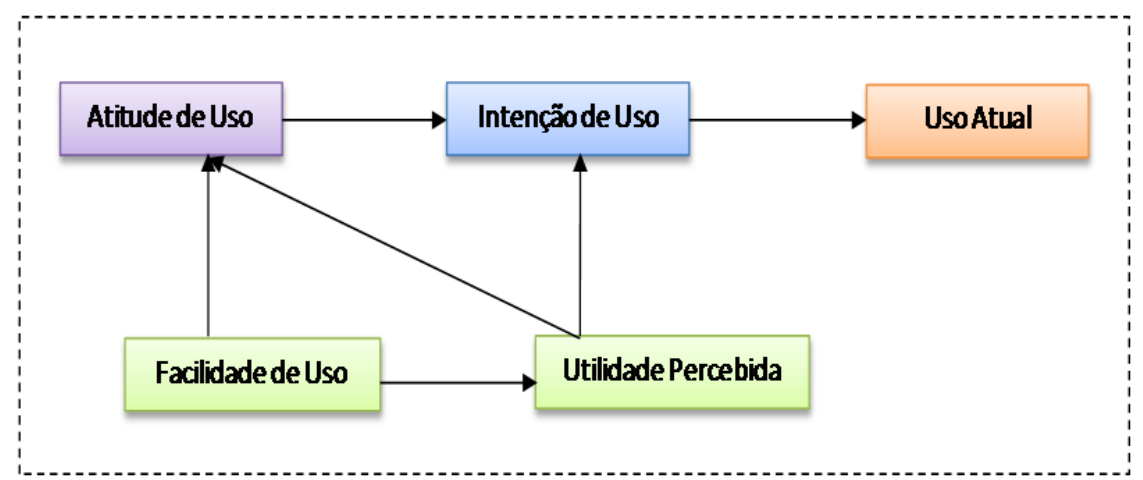

Figura 1: Modelo de aceitação da tecnologia (TAM)

Fonte: Adaptado de Dishaw e Strong (1999)

A utilidade percebida é influenciada pela facilidade de uso, e esses dois fatores interferem na atitude de uso da tecnologia (Löbler, Bobsin, Visentini e Vieira, 2010). A intenção de uso é desenvolvida pela atitude e pela utilidade e compreende o desejo dos indivíduos em usar a tecnologia, identificando se as pessoas têm preferência por usar os sistemas baseados em computador ao invés de métodos manuais (Dishaw e Strong, 1999).

Davis et al. (1989) ainda salientam a praticidade do modelo ao avaliar os SI, guiando os gestores a reduzir o problema da não utilização dos sistemas. Os autores ainda defendem a ideia de que os sistemas de informação não podem melhorar a performance da organização se não forem utilizados.

\subsection{O Modelo TTF (Task-Technology Fit) - Modelo de Ajuste Tecnologia-Tarefa}

A interdependência entre tecnologia da informação e desempenho individual tem sido uma preocupação nas pesquisas de sistemas de informação (Goodhue e Thompson, 1995). Assim, uma maneira de avaliar o comportamento de uso de uma tecnologia pode ser por meio de um modelo teórico. Neste caso, o Modelo de Ajuste Tecnologia-Tarefa (TTF), Figura 2, proposto por Goodhue e Thompson (1995) é amplamente empregado para avaliação do comportamento de uso de sistemas de informação (Dishaw, Strong e Bandy, 2002). Especificamente, o TTF é a correspondência entre exigências da tarefa, habilidades individuais e a funcionalidade da tecnologia (Goodhue e Thompson, 1995).

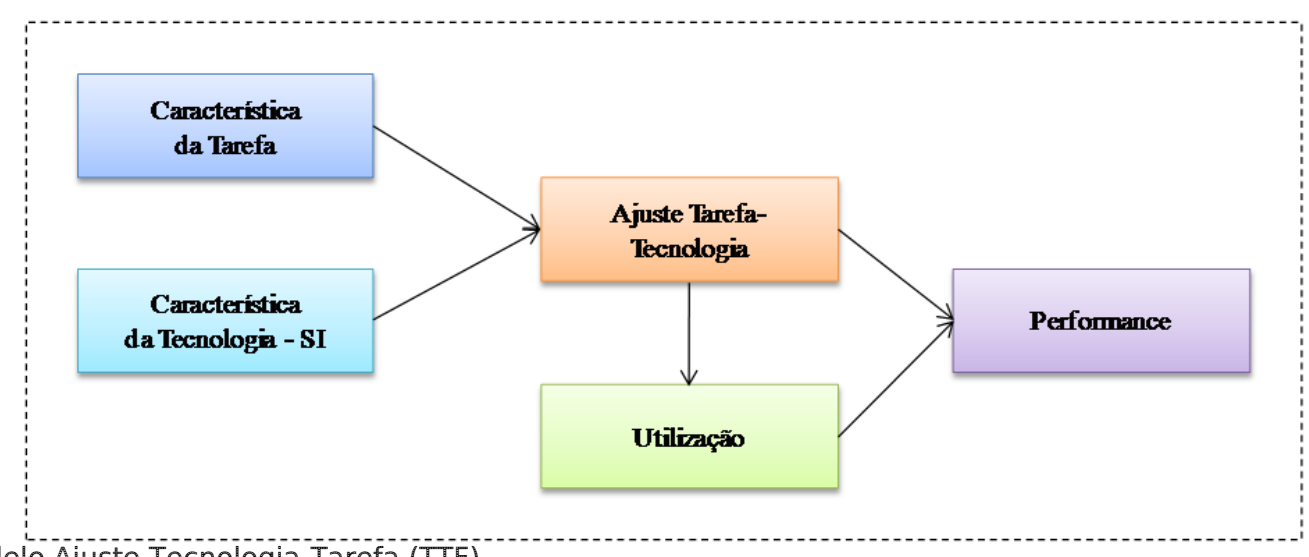

Figura 2: Modelo Ajuste Tecnologia-Tarefa (TT⿰亻

Fonte: Adaptdo de Dishaw e Strong (1999).

O modelo TTF indica que o desempenho do indivíduo é influenciado pelo ajuste existente entre as tarefas que ele realiza e a funcionalidade do SI, ou seja, quanto mais de acordo com as tarefas realizadas o sistema estiver, melhor será o desempenho do indivíduo (Goodhue e Thompson, 1995). Sob a ótica desses autores, o impacto do TTF na utilização ocorre a partir do vínculo de adequação entre tarefa e tecnologia e das crenças sobre as consequências de se usar um sistema tecnológico. 
Os principais construtos do modelo são os requerimentos das tarefas e a funcionalidade da ferramenta. De acordo com Santos (2004), tarefas são definidas como as ações que os indivíduos realizam para transformar entradas em saídas de modo a satisfazer as suas necessidades de informação. Assim, as características do indivíduo (conhecimento, habilidade, motivação) podem afetar a facilidade e o modo como se utiliza um sistema de informação. As tecnologias são ferramentas (hardware, software e dados) usados por indivíduos na realização das suas tarefas, e os fatores da tecnologia (acessibilidade, tempo de resposta) podem afetar a utilização. Nesse sentido, o TTF é a correspondência entre requisitos das tarefas, capacidades individuais e a funcionalidade de um sistema de informação (Santos, 2004).

O foco do TTF é o pressuposto de que os sistemas de informação são importantes por serem essenciais em algumas tarefas ou conjunto de tarefas e que os usuários irão refletir isso em suas avaliações sobre os sistemas (Goodhue, 1998). Assim, a mais forte ligação entre os SI e o desempenho será devida a uma correspondência entre a necessidade da tarefa e a funcionalidade do sistema (TTF) (Goodhue, 1998).

Para Goodhue e Thompson (1995), a experiência real de utilizar um SI pode levar os usuários a concluir que o si tem um melhor (ou pior) impacto sobre o desempenho do que o previsto, mudando suas consequências de utilização e consequentemente afetando a utilização futura. O indivíduo pode também aprender, a partir da experiência, a melhor maneira de utilizar o sistema, melhorando o ajuste individual deste e, portanto, o TTF global.

Ainda, segundo Dishaw e Strong (1999), esse modelo demonstra que o SI será usado exclusivamente se as opções fornecidas pelo sistema sustentarem as atividades do usuário. Caso contrário, os usuários escolherão ferramentas e métodos que Ihes permitam complementar a tarefa da melhor forma.

\section{Metodologia}

Visando atender aos objetivos delineados neste estudo, realizou-se uma pesquisa de natureza qualitativa, de cunho descritivo. No entendimento de Triviños (1987), estudos descritivos têm por objetivo descrever com precisão fatos e fenômenos, além disso, tais estudos visam à descrição das características de determinada população ou fenômeno ou então o estabelecimento de relações entre variáveis (Gil, 2002).

No que diz respeito ao método para a realização deste estudo, utilizou-se o estudo de caso, pois, conforme afirma Yin (2001), este tipo de estudo visa à investigação de um fenômeno contemporâneo dentro do contexto atual. O estudo de caso se caracteriza pelo estudo profundo e exaustivo de determinado fato ou fenômeno (GiL, 2002).

Este estudo teve seu foco de análise, dirigido a uma organização pertencente ao setor público, localizada na região central do estado do Rio Grande do Sul. A escolha da organização estudada foi do tipo intencional ou seleção racional (Barros e Lehfeld, 2004). Esta escolha se justifica pela importância dessa organização para a comunidade local, por prestar serviços de utilidade pública indispensáveis e pela responsabilidade que assume perante a sociedade.

O roteiro das entrevistas foi elaborado à luz do referencial teórico abordado, tendo por base o modelo TAM, proposto por Davis (1989), e o modelo TTF de Goodhue e Thompson (1995). Visando garantir a consistência do protocolo de entrevista, foram utilizados como base, os vários estudos já realizados de maneira quantitativa. O protocolo de entrevistas foi analisado e avaliado por um especialista em Sistemas de Informação. As categorias analíticas utilizadas para o alcance dos objetivos propostos são apresentadas de forma resumida na Figura 3.

Foram realizadas sete entrevistas semiestruturadas com pessoas que ocupam posições estratégicas na organização objeto do presente estudo, considerando que as mesmas utilizam o sistema como apoio ao processo decisório. Segundo Minayo (1996), a representatividade da pesquisa qualitativa não atém aos aspectos de ordem numérica, mas, sobretudo, busca-se abranger a totalidade do problema, elegendo, para tanto, indivíduos cuja relevância em relação a este problema seja identificada. As entrevistas foram gravadas e transcritas, sendo que, para garantir a confidencialidade das informações e preservar a identidade dos entrevistados, optou-se por nomeá-los de A a G.

Para a análise das entrevistas, foi utilizada a técnica da análise de conteúdo, proposta por Bardin (1977). Segundo a autora, a análise de conteúdo consiste em um conjunto de técnicas de análise das comunicações visando obter, por procedimentos sistemáticos e objetivos de descrição do conteúdo das 
mensagens (quantitativos ou não) que permitam a inferência de conhecimentos relativos às condições de produção/recepção (variáveis inferidas) destas mensagens. Segundo o entendimento de Minayo (1996), a análise de conteúdo pode abranger as seguintes etapas: pré-análise, onde ocorre a organização do material a ser analisado e definição das unidades de registro, unidades de contexto, trechos significativos e categorias; exploração do material, que consiste basicamente na operação de codificação, ou seja, a transformação dos dados brutos para o alcance do núcleo de compreensão do texto; e, tratamento dos resultados obtidos e interpretação, onde os resultados são submetidos a operações estatísticas simples ou complexas e, a partir disso, propõem-se inferências e realizam-se interpretações. No que tange às categorias de análise, estas foram definidas de acordo com o exposto na Figura 3.

\begin{tabular}{|c|c|c|c|}
\hline Fatores & Categorias & Descrição & Autores \\
\hline \multirow{4}{*}{$\begin{array}{l}\text { Utilidade } \\
\text { Percebida }\end{array}$} & Desempenho & $\begin{array}{l}\text { Contribuição do sistema para o } \\
\text { desempenho do trabalho. }\end{array}$ & \multirow{4}{*}{$\begin{array}{c}\text { Davis (1989, 1993); Davis e Venkatesh } \\
\text { (1996) }\end{array}$} \\
\hline & Produtividade & $\begin{array}{l}\text { Influência do sistema sob a } \\
\text { produtividade do trabalho. }\end{array}$ & \\
\hline & Rapidez & $\begin{array}{l}\text { O sistema possibilita a realização das } \\
\text { tarefas mais rapidamente. }\end{array}$ & \\
\hline & Utilidade & $\begin{array}{l}\text { Utilidade do sistema na realização das } \\
\text { tarefas. }\end{array}$ & \\
\hline \multirow{2}{*}{$\begin{array}{l}\text { Facilidade de } \\
\text { Uso }\end{array}$} & $\begin{array}{l}\text { Facilidade } \\
\text { Percebida }\end{array}$ & Facilidade em utilizar o sistema. & \multirow{2}{*}{$\begin{array}{c}\text { Dishaw e Strong (1999); Davis (1989, } \\
\text { 1993); Davis e Venkatesh (1996) }\end{array}$} \\
\hline & Uso Gerencial & $\begin{array}{c}\text { Facilidade de uso do sistema com fins } \\
\text { gerenciais. }\end{array}$ & \\
\hline \multirow{3}{*}{ Intenção de Uso } & $\begin{array}{l}\text { Intenção em } \\
\text { utilizar o sistema }\end{array}$ & $\begin{array}{c}\text { Intenção do usuário em utilizar o } \\
\text { sistema em substituição ou como uma } \\
\text { forma de complemento aos métodos } \\
\text { manuais. }\end{array}$ & \multirow{3}{*}{$\begin{array}{c}\text { Dishaw e Strong (1999); Davis (1989, } \\
\text { 1993); Davis e Venkatesh (1996) }\end{array}$} \\
\hline & Melhoria & $\begin{array}{c}\text { O usuário acredita que é melhor utilizar } \\
\text { o sistema do que outros métodos } \\
\text { manuais. }\end{array}$ & \\
\hline & $\begin{array}{l}\text { Gostar do } \\
\text { sistema }\end{array}$ & $\begin{array}{l}\text { O usuário gosta de utilizar o sistema } \\
\text { para a realização das tarefas. }\end{array}$ & \\
\hline \multirow{5}{*}{$\begin{array}{l}\text { Ajuste } \\
\text { Tecnologia- } \\
\text { Tarefa }\end{array}$} & $\begin{array}{l}\text { Detalhes das } \\
\text { informações }\end{array}$ & $\begin{array}{c}\text { Sistema oferece informações } \\
\text { detalhadas para a realização das } \\
\text { tarefas. }\end{array}$ & \multirow{5}{*}{$\begin{array}{c}\text { Dishaw e Strong (1999); Klopping e } \\
\text { McKinney (2004); } \\
\text { Goodhue e Thompson (1995); } \\
\text { Goodhue(1998) }\end{array}$} \\
\hline & $\begin{array}{l}\text { Localização das } \\
\text { informações }\end{array}$ & $\begin{array}{c}\text { Informações oferecidas pelo sistema } \\
\text { são de fácil localização. }\end{array}$ & \\
\hline & $\begin{array}{l}\text { Atualidade das } \\
\text { informações }\end{array}$ & $\begin{array}{l}\text { As informações fornecidas pelo SI são } \\
\text { atuais. }\end{array}$ & \\
\hline & $\begin{array}{l}\text { Compreensão e } \\
\text { Clareza }\end{array}$ & $\begin{array}{l}\text { O sistema fornece informações de fácil } \\
\text { compreensão e clareza. }\end{array}$ & \\
\hline & Confiabilidade & $\begin{array}{l}\text { O sistema fornece informações } \\
\text { confiáveis. }\end{array}$ & \\
\hline
\end{tabular}

Figura 3: Categorias analíticas

Fonte: Elaborado com base nos autores pesquisados

De acordo com os fatores que determinam o uso do SI, a partir dos modelos empregados neste estudo, a Figura 4 sintetiza o modelo de pesquisa utilizado.

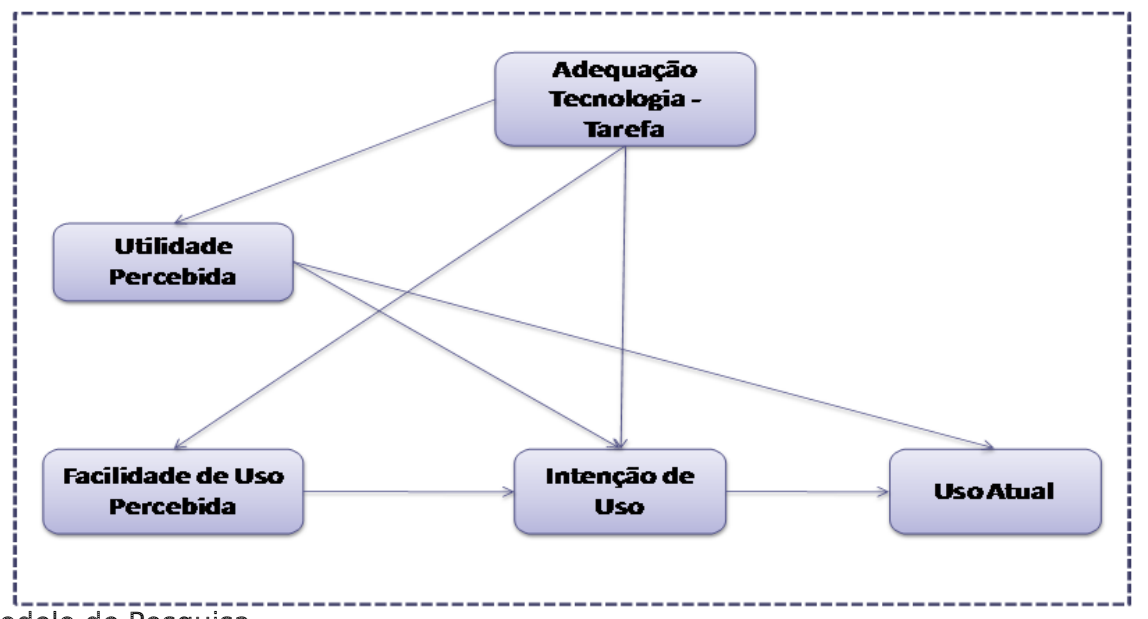

Figura 4: Modè̄o de Pesquisá

Fonte: Adaptado de Klopping e McKinney, 2004 


\section{Resultados}

\subsection{O Caso}

O caso estudado trata-se de uma empresa pública de segurança, de responsabilidade estadual. Por ser uma organização estatal centenária, possui características e especificidades em sua história e estrutura organizacional, as quais Ihe conferem um caráter ímpar. A instituição pesquisada possui como finalidades a preservação da ordem pública, incolumidade das pessoas e do patrimônio. Assim, é de sua competência atuar preventivamente, através de policiamento ostensivo, em locais ou áreas específicas do município, nos quais presume-se haver maior probabilidade de perturbação da ordem pública.

Nesse sentido, o foco do estudo residiu na análise de um Sistema de Informações Gerencial (SIG). O sistema estudado tem como finalidade gerir os dados relacionados à segurança pública a partir de relatórios. O sistema tem como fonte de informações as ocorrências, as quais são inseridas por indivíduos autorizados.

Foram entrevistados funcionários ligados à alimentação do sistema e utilização deste como fonte de relatórios gerenciais. Estes, por sua vez, estão alocados no primeiro e segundo escalão da instituição. A fim de manter a confidencialidade dos pesquisados, bem como preservar a instituição objeto de estudo, os mesmos não serão identificados em relação ao seu cargo.

\subsection{Fatores que explicam o uso de um Sistema de Informação na percepção dos entrevistados}

Com o objetivo de determinar quais os fatores que explicam o uso de um sistema de informação sob a ótica dos usuários que ocupam posições estratégicas na organização, foram avaliadas quatro dimensões: utilidade percebida, facilidade de uso, intenção de uso e ajuste tecnologia-tarefa. Essas dimensões, de acordo com o entendimento de Dishaw e Strong (1999) e Klopping e McKinney (2004), possibilitam uma maior compreensão dessa temática, pois reúne dois dos principais modelos utilizados para explicar o uso de um SI.

A utilidade percebida, conforme Davis (1993, p. 477), significa "o grau com que uma pessoa acredita que o uso de um sistema específico poderia melhorar a seu desempenho no trabalho". Nesse sentido, observou-se que os entrevistados foram unânimes em afirmar que o sistema apresenta contribuições em termos de desempenho e produtividade, como pode ser evidenciado a partir das seguintes falas:

Contribui inclusive, (...) que é para de repente quando a gente tem um decréscimo na nossa produtividade, através daí a gente pode identificar lá, determinado setor da unidade que a gente não está tendo a produtividade muito boa, até mesmo o servidor (...) que não esta produzindo. Porque tudo que é produzido é lançado no sistema, então tem um momento de verificar e procurar melhorar isso aí. (Entrevistado D)

É um mecanismo de mensurar essa produtividade(...), pois, tendo um mecanismo para mensurar (...), certamente influencia (...) hoje o ganho maior é justamente nessa quantificação e qualificação do nosso trabalho(...)ele pode ser melhorado em cima desse sistema (Entrevistado A).

De acordo com Davis (1989) e Davis e Venkatesh (1996), além do desempenho, o tempo empregado para a realização das tarefas exerce importante função para determinar a utilidade percebida, pois os usuários poderão imbuir utilidade a sistemas que possibilitem que suas atividades sejam desenvolvidas mais rapidamente. Nesse sentido, os entrevistados afirmaram que o sistema não tem contribuído significativamente para que as tarefas sejam realizadas mais rapidamente, conforme a fala a seguir.

Não vejo muita contribuição para rapidez no serviço. Mais para planejamento. (Entrevistado D)

Esse aspecto merece destaque, pois, embora afirmando que o sistema auxilia no desempenho e na produtividade do trabalho realizado, o mesmo não foi evidenciado no que diz respeito à rapidez com que os usuários executam as suas tarefas diárias. Contudo, os entrevistados, de maneira geral, percebem o sistema como útil para a realização do trabalho, embora o mesmo não seja imprescindível. Isso pode estar relacionado ao fato de que os entrevistados salientam que o sistema em questão é um sistema de apoio a tomada de decisão. Tais fatos podem ser evidenciados nas falas a seguir.

Eu não posso dizer imprescindível, pois eu acabei de dizer que a gente não tem uma rotina, um compromisso diário com o sistema, mas que ele é de bastante relevância é (...) ou talvez, dependendo até de uma posição de estratégia (...), ser adotada como prioridade, trabalhar baseado no sistema (Entrevistado D)

Ele traz informações que podem ser verificadas e que podem ser comparadas com outros sistemas para uma tomada de decisão. (Entrevistado B) 
Quanto à dimensão facilidade de uso percebida, esta pode ser entendida como o grau com que um individuo, utilizando um sistema, acredita que estará livre de esforços físicos ou mentais (DAVIS, 1989) . Dessa forma, os resultados deste estudo mostram que seis, dos sete entrevistados, destacaram que o sistema é de fácil utilização.

(...) não tenho dificuldade em trabalhar, eu acredito que seja um sistema fácil (...) me adaptei tranquilamente. (Entrevistado C)

Não ele não é muito fácil. Ele tem um número grande de janelas, e essas (...), à medida que tu vais abrindo janelas(...)então esse grande número de janelas causa alguma confusão(...)(Entrevistado B)

Evidenciou-se também, quanto à facilidade de uso para fins gerenciais, que o sistema contribui de maneira significativa para a organização estudada, na medida em que apresenta informações que auxiliam o planejamento e o processo de tomada de decisões estratégicas, como observado nas falas a seguir.

Nos deram um excelente subsídio para nossas ações (...), por nós termos as informações disponíveis ali..quando elas forem alimentados com tempo (Entrevistado F)

(...) é que ele é um sistema que é feito para comando (...) não é feito para a execução (Entrevistado G)

É, ele retrata (...) exatamente (...) por locais de maior incidência, turno, horários, exatamente isso (...) dá subsídios, ele traz o subsídio para que se planeje em cima (Entrevistado A)

Conforme Davis (1989), o usuário terá intenção de utilizar o sistema se o mesmo perceber as dimensões de utilidade e facilidade de uso. Nessa mesma linha, Dishaw e Strong (1999) enfatizam que um determinado sistema de informação será utilizado pelos usuários se o mesmo sustentar suas atividades, caso contrário, os usuários escolherão ferramentas e métodos que Ihes permitam complementar a tarefa da melhor forma.

Nesse sentido, todos os entrevistados afirmaram que o sistema é utilizado como complemento a outros métodos manuais, sendo que ainda não é possível substituí-los totalmente. Contudo, pode-se perceber que, embora ainda exista a necessidade de utilizar outros métodos manuais, os entrevistados assinalam que preferem utilizar o sistema a outras alternativas, fato que pode se comprovado através das falas a seguir.

Olha, eu acredito que ele seja um complemento na medida em que há determinados métodos manuais de que não se pode abrir mão(...)documentos operacionais. (Entrevistado A)

Eu considero o sistema como um complemento. Ele não chega a ser substituição. Porque tem que ter aquele primeiro controle ali (...) para lançar (...) tem que ser (...) é manual. (Entrevistado C)

Além disso, seis dos entrevistados, revelaram gostar do sistema, e apenas um afirmou que não gosta.

Eu gosto, mas é como eu disse, não é uma prioridade pra gente em virtude das outras atividades da gente. O ideal é que a gente pudesse verificar todo o dia. Eu, por exemplo, ainda tenho acesso a outros sistemas de informação que eu procuro olhar também (Entrevistado D)

Com relação à dimensão de ajuste da tecnologia à tarefa dos usuários, segundo Goodhue e Thompson (1995), ela é determinada pela correspondência entre as exigências da tarefa, habilidades individuais e a funcionalidade da tecnologia. Nesta mesma linha, Klopping e McKinney (2004) apontam que esse ajuste pode ser avaliado por meio do detalhamento, acessibilidade, atualidade, clareza e confiabilidade das informações fornecidas pelo sistema.

No que se refere ao nível de detalhamento das informações fornecidas pelo sistema em questão, os resultados demonstram que todos os entrevistados relataram que o sistema não fornece dados ricos em detalhes para a realização das tarefas.

Ele é mais genérico; não é muito detalhado (Entrevistado D)

Uma situação que é imprescindível para nós é saber o fato que ocorre por logradouro, por faixa de horário, por dia da semana, e essa informação a gente não tem (Entrevistado B)

Contudo, percebe-se que esse é um ponto que poderia ser mais bem explorado no sistema em questão, pois, considerando que o mesmo é utilizado para apoio à tomada de decisões, e que informações ricas em detalhes são consideradas como de qualidade (FISHER, CHENGALUR-SMITH, \& BALLOU, 2003), a riqueza em detalhes seria um aspecto importante, na medida em que poderia vir a auxiliar o processo decisório.

Quando questionados se as informações são de fácil localização no sistema, os entrevistados responderam que apresentam dificuldade em acessá-las. Entretanto, cabe ressaltar que os dados revelaram que isto depende da destreza e da experiência dos usuários, o que pode ser confirmado através da fala a seguir. 
Para quem já está habituado com o sistema sim. Para quem não está, vejo que o pessoal tem dificuldade (Entrevistado D)

Sobre a atualidade das informações fornecidas, seis dos entrevistados afirmaram que os mesmos são atuais, como pode ser evidenciado através das falas a seguir.

(...)vamos considerar por questão de segurança, 24horas. No dia é difícil. Tem alguns fatos que sim (Entrevistado F)

Atuais. Amanhã, às 8 horas da manhã já se sabe o que aconteceu (...) Aliás a partir da meia noite já se sabe o que aconteceu nas doze horas anteriores(...) (Entrevistado C)

Por outro lado, apenas um dos sujeitos entrevistados considera que as informações fornecidas pelo sistema não são suficientemente atuais, como pode se observar na fala a seguir.

Então assim, se hoje eu quiser uma estatística, ela não vai estar (...) não é uma coisa assim(...) automática (Entrevistado E)

Este fato merece destaque por tratar-se de uma organização do setor público e pela atualidade das informações; é de suma relevância para a comunidade local.

No que diz respeito à clareza das informações fornecidas pelo sistema, os entrevistados afirmaram que as informações fornecidas são de fácil compreensão e clareza conforme verificado na fala a seguir.

(...) depende tão exclusivamente da habilidade da pessoa em ler (...) então ele é bastante claro, bastante objetivo, bastante interativo (Entrevistado F)

Outro fator que merece destaque é a confiabilidade das informações. Nesse caso, os dados da pesquisa revelaram que, de um modo geral, as informações fornecidas são confiáveis, como pode ser confirmado pela fala do entrevistado A.

Elas são confiáveis na medida que elas estão com base na nossa rotina diária.

Vale a pena destacar que os entrevistados afirmam ter consciência da importância da alimentação do sistema para manter a confiabilidade dos dados, o que se pode confirmar pela fala do entrevistado D.

(...) depende muito da execução mesmo da corporação ter um compromisso com o sistema, porque se não tiver alimentação do sistema diário, das ocorrências todas lançadas ali, ela não vai ser confiável. Então a gente tem que ter realmente, tudo que for ocorrências detectadas na área da unidade devem ser inseridas ali para a gente ter um dado preciso.

Dessa forma, os resultados da pesquisa mostraram que, para o sistema em questão, todos os fatores estudados explicam o uso do SI. Evidencia-se, entretanto, que a categoria detalhamento da informação não foi percebida pelos usuários como significativa para explicar os fatores que os levam a utilizar o sistema.

\section{Considerações Finais}

A realização deste estudo possibilitou a confirmação, através de uma abordagem qualitativa, de dois modelos que, na maioria dos casos são aplicados em análises de natureza quantitativa para explicar o uso de um SI. Dessa forma, pode-se ampliar o entendimento acerca de tais modelos, visto que uma abordagem qualitativa pretende apenas entender a natureza de determinado fenômeno social (Richardson et al., 1985). É importante acrescentar a contribuição de Minayo e Sanches (1993) que entendem a abordagem qualitativa como uma aproximação fundamental e de identidade entre o sujeito e o objeto.

Assim, a presente pesquisa teve como objetivo identificar os fatores que explicam o uso de um sistema de informação. Além desse objetivo, o estudo apresentou objetivos secundários como: (i) identificar o uso de um SI através da facilidade, utilidade percebida e intenção de uso; e (ii) investigar o uso de um SI através do ajuste às tarefas.

No que se referem aos fatores que explicam o uso de um SI, de acordo com o modelo TAM, para o fator utilidade percebida, os dados da pesquisa mostraram que os entrevistados percebem que o sistema contribui para o seu desempenho e produtividade, embora eles acreditem que o sistema não tenha contribuído para uma maior agilidade na realização das tarefas. De forma geral, é considerado útil para o desenvolvimento do trabalho.

Acerca da facilidade de uso, os entrevistados, em sua maioria, afirmam que o sistema é de fácil utilização e ainda acrescentam que, para fins gerenciais, o sistema contribui de maneira significativa na definição de estratégias de ação para a organização. Sobre esses construtos (utilidade percebida e 
facilidade de uso), Davis (1989) enfatiza que os mesmos são de suma relevância para explicar a intenção de uso e consequentemente o uso de um sistema de informação.

No que tange ao construto ajuste da tecnologia à tarefa, os resultados sinalizam para uma convergência dos aspectos avaliados nas categorias facilidade de localização, clareza e compreensão, atualidade e confiabilidade dos dados, na medida em que todos se mostraram presentes no sistema estudado, exceto a categoria detalhamento das informações fornecidas. Dessa forma, pode-se dizer que há adequação entre o sistema e as tarefas desempenhadas, uma vez que, segundo Goodhue e Thompson (1995), o impacto do TTF na utilização de um sistema ocorre a partir do vínculo de adequação entre tarefa e tecnologia e das crenças sobre as consequências de usar um sistema tecnológico.

Por fim, observou-se que todos os fatores avaliados, utilidade percebida, facilidade de uso, intenção de uso e ajuste da tecnologia à tarefa foram os fatores que explicam o uso do sistema estudado. Dessa forma, percebe-se que o uso de um SI não está relacionado somente com o ajuste entre a tarefa e a tecnologia, ou com o comportamento e as atitudes dos indivíduos frente aos sistemas, mas também com a combinação desses fatores (Klopping e McKinney, 2004). Para uma melhor compreensão, a Figura 5 mostra a síntese dos resultados obtidos neste estudo.

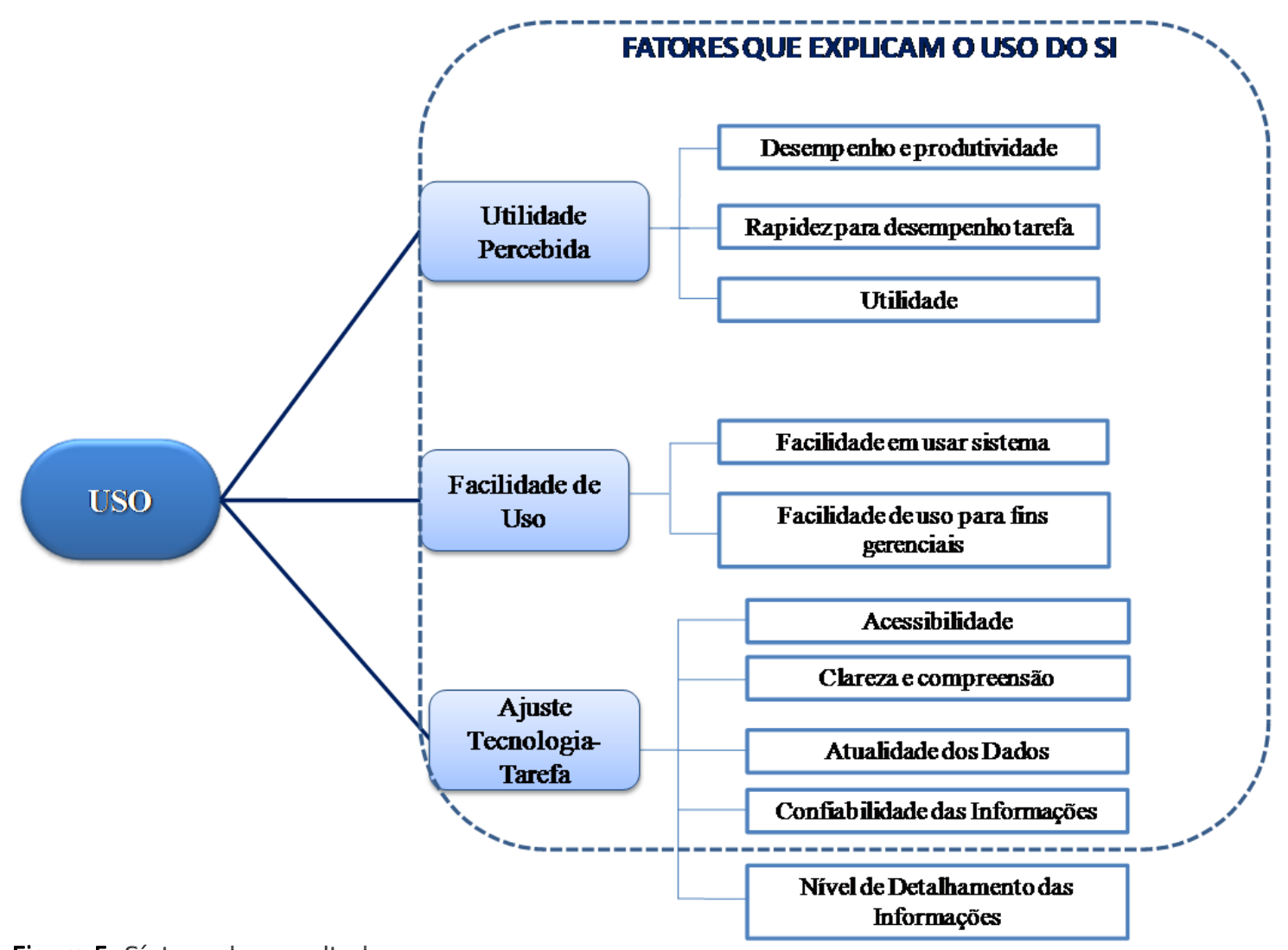

Figura 5: Síntese dos resultados Fonte: Elaborado pelos autores

Como limitações do estudo, pode-se citar o fato de que, por se tratar de um estudo de natureza qualitativa, o mesmo reflete apenas a percepção dos gestores entrevistados, além do fato de não se poder generalizar os resultados. Para pesquisas futuras, sugere-se a realização de estudos que combinem abordagens qualitativas e quantitativas.

\section{Referências}

Agarwal, R., Prasad, J. (1998). A Conceptual and Operational Definition of Personal Innovativeness in the Domain of Information Technology. Information System Research, 9 (2), 204-215.

Ajzen, I. (1985). From intentions to actions: A theory of planned behavior. In Kuhl, J.; Beckman, J. (ed.). Action control: From cognition to behavior. Heidelberg, Germany: Springer. 
Ajzen, I., Madden, T. (1986). Prediction of goal-directed behavior: attitudes, intentions, and perceived behavioral control. Journal of Experimental Social Psychology, 22, 453-474.

Ajzen, I. (1988). Attitudes, personality, and behavior. Milton Keynes: Open University Press. Audy, J. L. N., Andrade, G. K. de, Cidral, A. (2005). Fundamentos de sistemas de informação. Porto Alegre: Bookman.

Bardin, L. (1977). Análise de Conteúdo. Lisboa: Edições 70.

Barros, A. I. S., Lehfeld, N.A.S. (2004). Fundamentos de metodologia científica. São Paulo: Pearson Makron Books.

Chau, P. Y. K.; Hu, P. J-H. (2001). Information Technology Acceptance by Individual Professionals: A Model Comparison Approach. Decision Sciences, 32 (4), 699-719.

Davis, F. D. (1989). Perceived usefulness, perceived ease of use, and user acceptance of information technology. MIS Quarterly, 13 (3), 319-339.

Davis, F. D., Bagozzi, R. P., Warshaw, P. R. (1989). User acceptance of computer technology: a comparison of two theoretical models. Management Science, 35 (8), 982-1003.

Davis, F. D. (1993). Use acceptance of information technology: system characteristics, user perceptions and behavioral impacts. International Journal of Man-Machine Studies, 38, 475-487.

Davis, F. D., Venkatesh, V. (1996). A critical assessment of potential measurement biases in the technology acceptance model: three experiments. International Journal of Human-Computer Studies, 45 (1), 19-46.

Dishaw, M. T., Strong, D. M. (1999). Extending the technology acceptance model with task technology fit constructs. Information and Management, 36, p. 9-21.

Dishaw, M. T., Strong, D. M., Bandy, D. B. (2002). Extending the Task-Technology Fit Model with Self-Efficacy Constructs. In Eighth Americas Conference on Information Systems.

Fishbein, M., Ajzen, I. (1975). Belief, attitude, intention and behavior: an introduction to theory and research. Boston: Addison-Wesley.

Fisher, C. W., Chengalur-Smith, I., Ballou, D. P. (2003). The impact of experience and time on the use of data quality information in decision making. Information Systems Research, 14(2) 170189.

Gil, A. C. (2002). Como elaborar projetos de pesquisa. 4. ed. São Paulo: Atlas.

Goodhue, D. L. (1995). Understanding user evaluations of information systems. Management Science, 41 (12), 1827-1844.

Goodhue, D. L., Thompson, R. L. (1995). Task-technology fit and individual performance. MIS Quarterly, 19 (2), 213-236.

Goodhue, D. L. Development and measurement validity of a task-technology fit instrument for user evaluations of information systems. Decision Sciences, 29, 105-138, Winter 1998.

Igbaria, M., livari, J., Maragahh, H. (1995). Why do individuals use computer technology? A finnish case study. Information \& Management, 29, p. 227-238.

Klopping, I. M., Mckinney, E. Extending the Technology Acceptance Model and the TaskTechnology Fit Model to Consumer e-Commerce. Information Technology, Learning, and Performance Journal, 22 (1), 35.

Laudon, K. C.; Laudon, J. P. (2004). Sistemas de informações gerenciais: administrando a empresa digital. São Paulo: Prentice Hall.

Lee, Y.; Kozar, K. A., Larsen, K. R. T. (2003). The technology acceptance model: past, present, and future. Communications of the Association for Information Systems, 12, 752-780.

Minayo, M. C. de S., Sanches, O. (1993). Quantitativo-qualitativo: oposição ou complementaridade? Cad. Saúde Pública, 9 (3), 239-282, jul./set. 
Moresi, E. A. D. (2000). Delineando o valor do sistema de informação de uma organização. Ciência da Informação, 29 (1), 14-24, jan./abr.

O'Brien, J. A. (2004). Sistemas de informação e as decisões gerenciais na era da internet. São Paulo: Saraiva, 2. ed.

Oliveira, D. de P. R. (1993). Sistemas de informações gerenciais: estratégicas, táticas operacionais. 2. ed. São Paulo: Atlas.

Richardson, R. J. et al. (1985). Pesquisa social: métodos e técnicas. São Paulo: Atlas.

Stair, R. M., Reynolds, G. W. (2002). Princípios de sistemas de informações. Rio de Janeiro: LTC.

Triviños, A. N. S. (1987). Introdução à pesquisa em Ciências Sociais: a pesquisa qualitativa em educação. São Paulo: Atlas.

Turban, E., Rainer Jr., R. L., Potter, R. E. (2005). Administração de Tecnologia da Informação: Teoria e Prática. São Paulo, Campus.

Turban, E., Leidner, D., Mcclean, E., Wetherbe, J. (2006). Information Technology for management: transforming organizations in the digital economy. John Wiley \& Sons.

Vemkatesh, V., Bala, H. (2008). Technology Acceptance Model 3 and a Research Agenda on Interventions. Decision Sciences 39 (2), May.

Yin, R. (2001). Estudo de Caso: Planejamento e Métodos. Porto Alegre: Bookman.

Zhang, P., Li, N., Sun, H. (2006). Affective Quality and Cognitive Absorption: Extending Technology Acceptance Reserch. Proceedings of the Havai International Conference on System Science. 


\section{Nota de Errata}

Página mantida visando preservação da numeração dos artigos após efetuadas as seguintes correções de errata:

1) Alteração nas referências do artigo em função das citações no corpo do texto.

Errata realizada em 07/06/2012 por Diego Maganhotto Coraiola. 